\title{
NR2F2 loss-of-function mutation is responsible for congenital bicuspid aortic valve
}

\author{
JUAN WANG $^{1 *}$, PRADHAN ABHINAV ${ }^{1 *}$, YING-JIA XU $^{2 *}$, RUO-GU LI $^{3}$, MIN ZHANG $^{3}$, XING-BIAO QIU ${ }^{3}$, \\ RUO-MIN DI ${ }^{2}$, QI QIAO ${ }^{2}$, XIU-MEI LI ${ }^{2}$, RI-TAI HUANG ${ }^{4}$, SONG XUE $^{4}$ and YI-QING YANG ${ }^{2,5,6}$ \\ ${ }^{1}$ Department of Cardiovascular Medicine, East Hospital, Tongji University School of Medicine, Shanghai 200120; \\ ${ }^{2}$ Department of Cardiology, The Fifth People's Hospital of Shanghai, Fudan University, Shanghai 200240; \\ ${ }^{3}$ Department of Cardiology, Shanghai Chest Hospital, Shanghai Jiao Tong University, Shanghai 200030; \\ ${ }^{4}$ Department of Cardiovascular Surgery, Renji Hospital, School of Medicine, Shanghai Jiao Tong University, \\ Shanghai 200127; ${ }^{5}$ Cardiovascular Research Laboratory and ${ }^{6}$ Central Laboratory, \\ The Fifth People's Hospital of Shanghai, Fudan University, Shanghai 200240, P.R. China
}

Received July 20, 2018; Accepted January 22, 2019

DOI: $10.3892 /$ ijmm.2019.4087

\begin{abstract}
Congenital bicuspid aortic valve (BAV) represents the most common type of cardiac birth defect affecting $0.4-2 \%$ of the general population, and accounts for a markedly increased incidence of life-threatening complications, including valvulopathy and aortopathy. Accumulating evidence has demonstrated the genetic basis of BAV. However, the genetic basis for BAV in the majority of cases remains to be elucidated. In the present study, the coding regions and splicing donors/acceptors of the nuclear receptor subfamily 2 group $\mathrm{F}$ member 2 (NR2F2) gene, which encodes a transcription factor essential for proper cardiovascular development, were sequenced in 176 unrelated cases of congenital BAV. The available family members of the proband carrying an identified $N R 2 F 2$ mutation and 280 unrelated, sex- and ethnicity-matched healthy individuals as controls were additionally genotyped for $N R 2 F 2$. The functional effect of the mutation was characterized using a dual-luciferase reporter assay system. As a result, a novel heterozygous $N R 2 F 2$ mutation, NM_021005.3: c.288C>A; p.(Cys96*), was identified in a family with BAV, which was transmitted in an autosomal
\end{abstract}

Correspondence to: Dr Juan Wang, Department of Cardiovascular Medicine, East Hospital, Tongji University School of Medicine, 150 Jimo Road, Shanghai 200120, P.R. China

E-mail: wang_juan986@163.com

Dr Yi-Qing Yang, Cardiovascular Research Laboratory, The Fifth People's Hospital of Shanghai, Fudan University, 801 Heqing Road, Shanghai 200240, P.R. China

E-mail: yangyiqing@5thhospital.com

${ }^{*}$ Contributed equally

Key words: congenital heart disease, bicuspid aortic valve, genetics, transcription factor, nuclear receptor subfamily 2 group F member 2, reporter gene assay dominant mode with complete penetrance. The nonsense mutation was absent from the 560 control chromosomes. Functional analysis identified that the mutant NR2F2 protein had no transcriptional activity. Furthermore, the mutation disrupted the synergistic transcriptional activation between NR2F2 and transcription factor GATA-4, another transcription factor that is associated with BAV. These findings suggested $N R 2 F 2$ as a novel susceptibility gene of human BAV, which reveals a novel molecular pathogenesis underpinning BAV.

\section{Introduction}

As the most common cardiac developmental malformation in humans, congenital bicuspid aortic valve (BAV) affects $0.5-2 \%$ of the Western population (1) and $\sim 0.4 \%$ of the Chinese population (2), and has a higher incidence in patients with other congenital cardiac abnormalities $(3,4)$. Although it is commonly diagnosed by echocardiography in asymptomatic cases (1), approximately one-third of all BAV cases develop various complications in their lifetime, encompassing aortic stenosis, regurgitation, dilatation, dissection, aneurysm and rupture, in addition to infective endocarditis, hypoplastic left ventricle, ventricular fibrosis, heart failure, cardiac arrhythmias and sudden cardiac death (5-9). BAV is responsible for $70-85 \%$ of cases of aortic stenosis in children and $>50 \%$ of cases of aortic stenosis in adults (10). Furthermore, aortic dilatation occurs in $30-70 \%$ of patients with BAV, and aortic valve dysfunction occurs in $\leq 47 \%$ of patients with BAV (11). BAV is also associated with an eight-fold increased risk of aortic dissection, and over a 25 -year period, the risks for aortic valve replacement, aortic aneurysm occurrence and aortic surgery are 53, 26 and 25\%, respectively (10). Therefore, BAV may lead to severe health complications when compared with all other congenital heart defects combined $(10,11)$. Despite important clinical significance, the etiologies underlying BAV remain undetermined.

During fetal development, cardiac valvular morphogenesis is understood to begin with the formation of endocardial 
cushions, the precursors to mature cardiac valves. It subsequently undergoes a complex developmental process that orchestrates the coordination of cell proliferation, differentiation, migration, adhesion and apoptosis; genetic and environmental pathogenic factors may disturb this process, contributing to abnormal valvulogenesis and the pathogenesis of BAV (10-13). However, accumulating evidence suggests the strong genetic basis of BAV, particularly for familial BAV, and mutations in $>10$ genes, encompassing those coding for extracellular matrix proteins, signaling molecules and cardiac transcription factors, including GATA transcription factors GATA4-6 and homeobox protein NKX2-5, which are associated with BAV in humans (13-24). As another cardiac core transcription factor, nuclear receptor subfamily 2 group $\mathrm{F}$ member 2 (NR2F2) is expressed at high levels in the embryonic heart and aorta, and serves an essential role in cardiac and valvular morphogenesis in vertebrates $(25,26)$. Furthermore, the expression and functional profile of $N R 2 F 2$ overlap at least partially with those of the well-established BAV-associated genes GATA4, GATA5 and NKX2-5 during embryonic cardiogenesis (27-29). Pathogenic mutations in NR2F2, GATA4-6 and $N K X 2-5$ have been reported to cause similar cardiovascular developmental anomalies in animals and humans, including defects in the cardiac outflow tract, septum and endocardial cushion (30-40). These findings suggest $N R 2 F 2$ as a preferred candidate gene of human BAV and provide a theoretical basis for screening of the gene. The present study aimed to identify novel $N R 2 F 2$ mutations associated BAV and the underlying mechanism by which mutant $N R 2 F 2$ contributes to BAV.

\section{Materials and methods}

Study subjects. Between January 2015 and February 2018, a study cohort of 176 unrelated cases diagnosed with BAV was enlisted from the Chinese Han-ethnic population. The available relatives of the index patient carrying an identified $N R 2 F 2$ mutation were additionally recruited. A control cohort of 280 unrelated healthy subjects without BAV (170 men and 110 women, with a mean age of $45 \pm 10$ years), matched with patients with BAV for age, sex and ethnicity, was enrolled from the same geographical area. The sample size was estimated according to the consensus that the prevalence of the genetic mutation is $<1.0 \%$ among the general population, with $95 \%$ confidence and a margin of error of $<0.02$. The individuals recruited to the present study underwent comprehensive clinical appraisal, encompassing familial and medical histories, detailed physical examination, and two-dimensional echocardiography. In each patient, a diagnosis of BAV was made according to echocardiographic images and/or surgical records of aortic valve replacement surgery (2). Cases with recognized chromosomal abnormalities or other syndromes, including Turner syndrome and DiGeorge syndrome, were excluded from the investigation at the time of enrollment. The present study was conducted in conformity with the ethical principles described by the Declaration of Helsinki. The study protocol was approved by the Medical Ethics Committee of Dongfang Hospital, Tongji University (Shanghai, China). Whole peripheral venous blood samples and clinical data were collected from the study subjects subsequent to providing written informed consent to participate in the investigation.
Mutational analysis of NR2F2. Genomic DNA samples were isolated from venous blood leukocytes with the QIAamp DNA Blood Mini kit (Qiagen GmbH, Hilden, Germany) following the manufacturer's manual. Direct polymerase chain reaction (PCR)-sequencing and sequence analysis of the coding regions and splicing donors/acceptors of the $N R 2 F 2$ gene were performed in the patients with BAV and control individuals. PCR and the procedures of sequencing analysis were conducted as previously described (31). The identified sequence variation was retrieved in databases as previously described (41-43) to confirm its novelty. The databases included the single nucleotide polymorphism database (https://ncbi.nlm.nih. gov/projects/SNP), the exome aggregation consortium database version 0.3.1 (http://exac.broadinstitute.org/), the human gene mutation database (http://www.hgmd.cf.ac.uk/ac/index.php), the 1000 genome project database (http://www.internationalgenome.org/) and the exome variant server database version 2.0 (http://evs.gs.washington.edu/EVS).

Plasmids and site-targeted mutagenesis. The cDNAs were prepared from human heart samples as previously described (44-47). The wild-type NR2F2-pcDNA3.1 plasmid expressing human NR2F2 was constructed as previously reported (31). The mutation detected in patients with BAV was introduced into the wild-type NR2F2-pcDNA3.1 plasmid by site-directed mutagenesis with the QuickChange II XL Site-Directed Mutagenesis kit (Stratagene; Agilent Technologies, Inc., Santa Clara, CA, USA) with a complementary pair of primers (forward, 5'-CGGCCAGTTCACGTGAGAGGGCTG CAAGAGC-3'; reverse, 5'-GCTCTTGCAGCCCTCTCACGT GAACTGGCCG-3') following the manufacturer's protocol, and was verified by direct PCR sequencing. The expression plasmid GATA4-pSSRa, the apolipoprotein B (APOB) promoter-driven firefly luciferase (APOB-luc) reporter plasmid and the atrial natriuretic factor (ANF) promoter-driven firefly luciferase reporter plasmid (ANF-luc) used in the present study, were as previously described (31).

Cell culture, transient transfection and luciferase assay. COS-7 and 293 cells, the two cell lines used most in previous functional studies of NR2F2, were grown in Dulbecco's modified Eagle's medium (Gibco; Thermo Fisher Scientific, Inc., Waltham, MA, USA) with $10 \%$ fetal bovine serum (Invitrogen; Thermo Fisher Scientific, Inc.) in addition to $100 \mu \mathrm{g} / \mathrm{ml}$ streptomycin (Sigma-Aldrich; Merck KGaA, Darmstadt, Germany) and $100 \mathrm{U} / \mathrm{ml}$ penicillin (Sigma-Aldrich; Merck KGaA) in an incubator with an atmosphere of $5 \% \mathrm{CO}_{2}$ at $37^{\circ} \mathrm{C}$. The cells were seeded at a density of $1 \times 10^{5}$ cells/well in 12 -well plates $24 \mathrm{~h}$ prior to transfection with various plasmids using SuperFect transfection reagent (Invitrogen; Thermo Fisher Scientific, Inc.) according to the manufacturer's protocol. Additionally, as an internal control to normalize the transfection efficiency, the pGL4.75 plasmid (Promega Corporation, Madison, WI, USA) expressing Renilla luciferase was co-transfected into the cells. Cellular transfection experiments with various plasmids were performed as previously described (31). The cells were cultured at $37^{\circ} \mathrm{C}$ and lysed $36 \mathrm{~h}$ after transient transfection. The luciferase activity of the cellular lysates was measured using the Dual-Luciferase Reporter Assay system (Promega Corporation) following the manufacturer's protocol. 
Table I. Baseline clinical and valvular characteristics of the 176 cases with congenital BAV.

\begin{tabular}{|c|c|c|c|}
\hline Variable & Patient group $(\mathrm{n}=176)$ & Control group $(n=280)$ & P-value \\
\hline Age (years) & $45 \pm 12$ & $45 \pm 14$ & $\mathrm{P}>0.9999$ \\
\hline Male & $105(60)$ & $164(59 \%)$ & $\mathrm{P}=0.8182$ \\
\hline BMI $\left(\mathrm{kg} / \mathrm{m}^{2}\right)$ & $23 \pm 3$ & $23 \pm 4$ & $\mathrm{P}>0.9999$ \\
\hline Chronic heart failure (NYHA class $\geq \mathrm{II}$ ) & $126(72)$ & $0(0)$ & $\mathrm{P}<0.0001$ \\
\hline Positive family history of BAV & $39(22)$ & $0(0)$ & $\mathrm{P}<0.0001$ \\
\hline \multicolumn{4}{|l|}{ Classification of BAV morphology } \\
\hline $\mathrm{R}-\mathrm{L}$ & $116(66)$ & NA & NA \\
\hline $\mathrm{R}-\mathrm{NC}$ & $58(33)$ & NA & NA \\
\hline L-NC & $2(1)$ & NA & NA \\
\hline Aortic regurgitation & $103(59)$ & NA & NA \\
\hline Mild & $46(26)$ & NA & NA \\
\hline Moderate & $38(22)$ & NA & NA \\
\hline Severe & $19(11)$ & NA & NA \\
\hline Aortic stenosis & $65(37)$ & NA & NA \\
\hline Mild & $30(17)$ & NA & NA \\
\hline Moderate & $25(14)$ & NA & NA \\
\hline Severe & $10(6)$ & NA & NA \\
\hline Valve calcification & $71(40)$ & NA & NA \\
\hline Other concomitant congenital heart defects ${ }^{\mathrm{a}}$ & $16(9)$ & NA & NA \\
\hline Surgery or catheter-based therapy & $111(63)$ & NA & NA \\
\hline
\end{tabular}

Data are presented as the mean \pm standard deviation for continuous variables or as number $(\%)$ for categorical variables. Continuous variables (age and BMI) between the patient and control groups were compared using Student's unpaired t-test. Categorical variables of males between the patient and control groups was compared using Pearson's $\chi^{2}$ test, whereas other categorical variables (chronic heart failure and positive family history of BAV) between the two groups were compared using Fisher's exact test. ${ }^{a}$ Seven patients had ventricular septal defect, four had atrial septal defect, two had coarctation of the aorta, two had patent ductus arteriosus, and one had aortic stenosis. BAV, bicuspid aortic valve; BMI, body mass index; L-NC, fusion of left coronary cusp and non-coronary cusp; NA, not applicable or available; NYHA, New York Heart Association; R-L, fusion of right coronary cusp and left coronary cusp; R-NC, fusion of right coronary cusp and non-coronary cusp.

The transcriptional activity of the target gene promoters is presented as the fold activation of firefly luciferase relative to Renilla luciferase. For each plasmid, three independent transient transfection experiments were completed in triplicate and the results are presented as the mean \pm standard deviation.

Statistical analysis. Statistical analyses were performed using the SPSS for Windows statistical software package version 19.0 (IBM Corp., Armonk, NY, USA). Continuous variables, including age, body mass index and the transcriptional activity of the target gene promoters, are presented as the mean \pm standard deviation. Categorical variables, including sex, ethnicity, chronic heart failure, family history of BAV, classification of BAV morphologies and incidence of various BAV-associated clinical phenotypes, are presented as a number and percentage. Categorical variables were compared using Pearson's $\chi^{2}$ test (for sex and ethnicity) or Fisher's exact test (for chronic heart failure and family history of BAV), where appropriate. The comparison of continuous variables between two groups was conducted with Student's unpaired t-test. One-way analysis of variance, followed by Fisher's protected least-squares difference post hoc test, was used when multiple groups were compared. A two-sided $\mathrm{P}<0.05$ was considered to indicate a statistically significant difference.

\section{Results}

Clinical and valvular features of the present study participants. In the present study, 176 unrelated patients with BAV were clinically investigated, in addition to 280 unrelated healthy controls without BAV. The cases were matched with control subjects for ethnicity, sex and age. No significant difference in body mass index was found between the patient and control groups $(23 \pm 3$, vs. $23 \pm 4, \mathrm{P}=0.99998)$; whereas significant differences in the incidence of heart failure and family history of BAV were found between the two groups $(126 / 176$, vs. $0 / 280, \mathrm{P}<0.00001$ for heart failure; $39 / 176$, vs. $0 / 280, \mathrm{P}<0.00001$ for family history of BAV). All cases had echocardiogram-documented BAV. Of the BAV group, $\sim 66 \%(n=116), 33 \%(n=58)$ and $1 \%(n=2)$ had fusion of the left and right coronary, right and noncoronary, and left and noncoronary cusps, respectively. Coexistent cardiovascular malformations were present in $\sim 9 \%$ of cases of BAV, and these were predominantly cardiac septal defects. The control subjects' echocardiograms demonstrated normal cardiac images without evidence of structural cardiac deformities. The baseline clinical and valvular characteristics of the 176 unrelated BAV cases are summarized in Table I. 

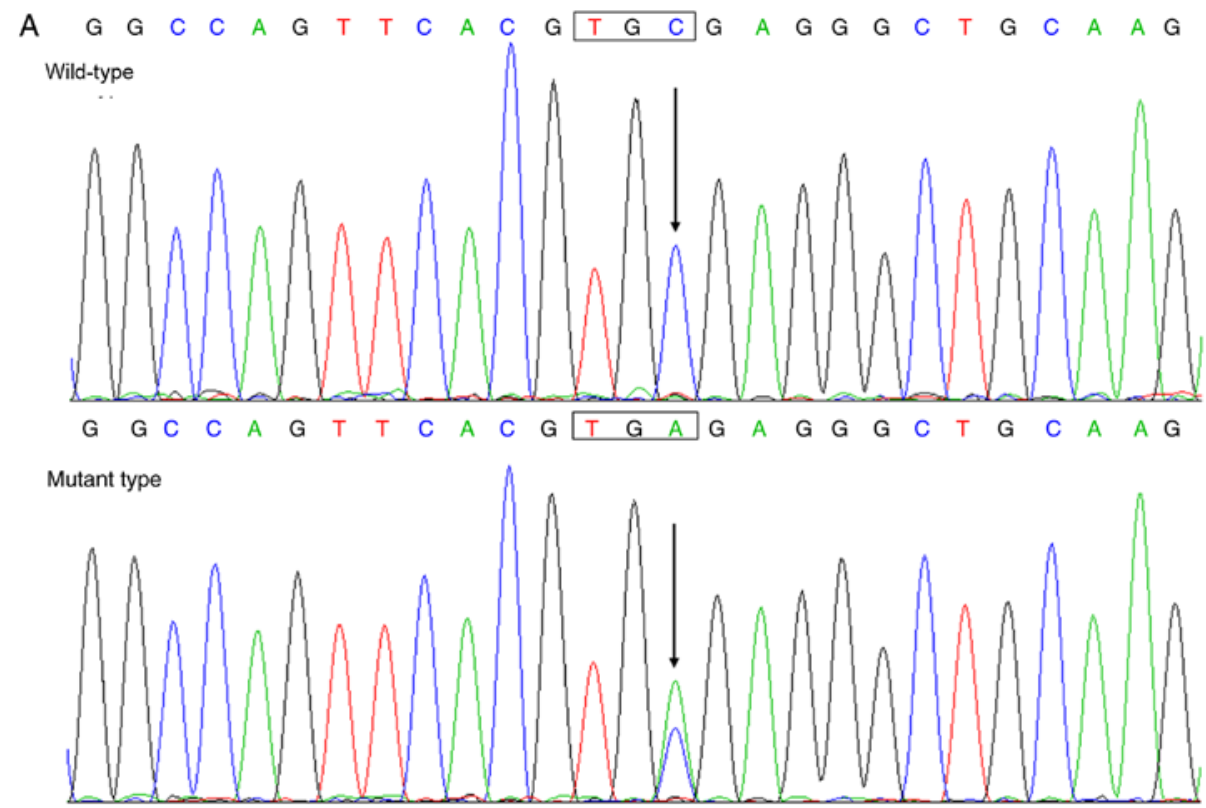

B

Wild-type
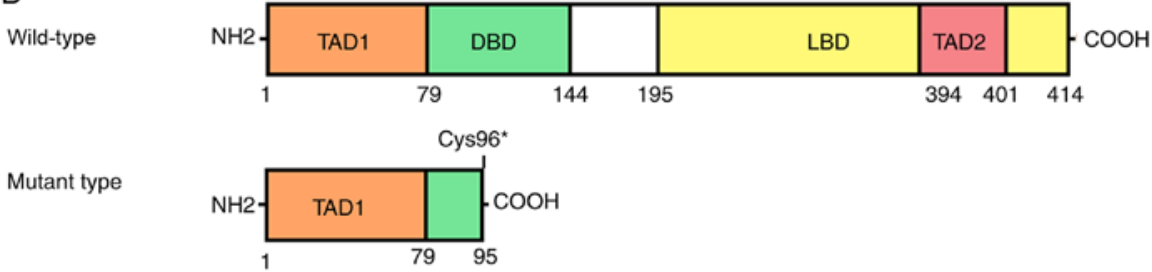

C Family 1

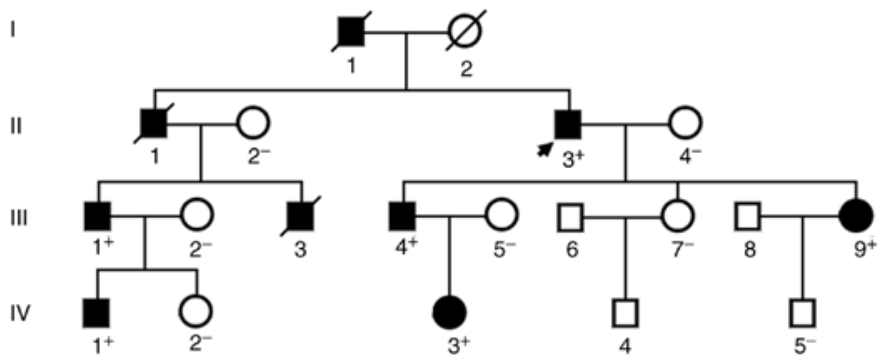

Figure 1. A novel NR2F2 mutation linked to congenital BAV. (A) Sequence chromatograms exhibiting the heterozygous $N R 2 F 2$ mutation and its homozygous wild-type control. The arrow points to heterozygous nucleotides of $\mathrm{C} / \mathrm{A}$ in the proband (mutant type) or homozygous nucleotides of $\mathrm{C} / \mathrm{C}$ in a control individual (wild-type). The rectangle marks the nucleotides comprising a codon of $N R 2 F 2$. (B) Schematic drawings illustrating the wild-type and Cys96-mutant NR2F2 protein structures. The mutation predisposing to congenital BAV is marked above the structural domains. (C) Pedigree of the family with congenital BAV. The family was arbitrarily designated as family 1 . Family members are identified by generations and numbers. Squares indicate male family members; circles indicate female members; closed symbols indicate affected members; open symbols indicate unaffected members; symbols with a slash indicate deceased members; the arrow adjacent to the closed square indicates the proband. +, carrier of the heterozygous mutation; -, non-carrier; NR2F2, nuclear receptor subfamily 2 group F member 2; BAV, bicuspid aortic valve; TAD1, transcriptional activation domain 1; DBD, DNA-binding domain; LBD, ligand binding domain; TAD2, transcriptional activation domain 2; $\mathrm{NH} 2$, amino terminus; $\mathrm{COOH}$, carboxyl terminus.

Identification of a novel NR2F2 mutation. Through mutational analysis of the coding exons and splicing signal sequences of the $N R 2 F 2$ gene in 176 unrelated cases of BAV, a heterozygous mutation was identified in an index patient aged 54 years. Specifically, a substitution of adenine (A) for cytosine (C) at the third nucleotide of codon 96 (NM_021005.3: c.288C >A), predicting alteration of the codon coding for cysteine (Cys) at amino acid position 96 to a premature stop codon, p.(Cys96*), was identified in a male with BAV, who was positive for a family history of BAV. The DNA sequence chromatograms showing the heterozygous c. $288 \mathrm{C}>\mathrm{A}$ mutation of $N R 2 F 2$ and its homozygous wild-type sequence are presented in Fig. 1A. The schematic drawings denoting the principal structural domains of wild-type and Cys96*-mutant NR2F2 proteins, in addition to the location of the mutation detected in the present investigation, are presented in Fig. 1B. The identified mutation was not observed in the 280 control individuals, nor was it reported in the databases described elsewhere (41-43). Mutational screening of the available relatives of the index patient revealed that the nonsense mutation was present in all affected relatives; however, this was absent in the unaffected relatives. Genetic analysis of the lineage of the proband identified that the nonsense mutation co-segregated with BAV, which was transmitted in an autosomal dominant pattern in the family, with complete penetrance. In this family, all family members affected with BAV had fusion of the right and 
Table II. Phenotypic features and status of the NR2F2 mutation of the living affected family members.

\begin{tabular}{lcclc}
\hline & & & \multicolumn{1}{c}{ Phenotype } & Genotype \\
Identity & Sex & Age (years) & Congenital heart defect & Cys96* \\
Family 1 & & & & $+/-$ \\
II-3 & M & 54 & BAV (R-L) & $+/-$ \\
III-1 & M & 32 & BAV (R-L), VSD & $+/-$ \\
III-4 & M & 30 & BAV (R-L) & $+/-$ \\
III-9 & F & 25 & BAV (R-L) & $+/-$ \\
IV-1 & M & 6 & BAV (R-L), VSD & $+/-$ \\
IV-3 & F & 4 & BAV (R-L) & \\
\hline
\end{tabular}

$N R 2 F 2$, nuclear receptor subfamily 2 group F member 2; BAV, bicuspid aortic valve; F, female; M, male; R-L, fusion of right coronary cusp and left coronary cusp; VSD, ventricular septal defect; +/-, heterozygote.

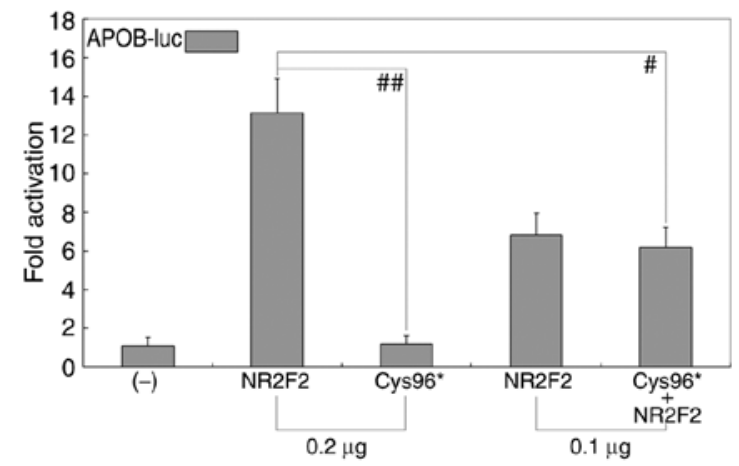

Figure 2. Functional failure of NR2F2 results from the mutation. Activation of the $A P O B$ promoter driven luciferase in 293 cells by wild-type NR2F2 or Cys96*-mutant NR2F2 (Cys96*), alone or in combination, showed no transcriptional activation by the mutant protein. Experiments were performed in triplicate and results are presented as the mean \pm standard deviation. ${ }^{\# \#} \mathrm{t}=11.2448, \mathrm{P}=0.00036$ and ${ }^{\#} \mathrm{t}=5.83662, \mathrm{P}=0.00430$, compared with wild-type NR2F2 $(0.2 \mu \mathrm{g})$. One-way analysis of variance, followed by the Fisher's protected least-squares difference post-hoc test, was used for multiple comparisons (among $0.2 \mu \mathrm{g}$ of wild-type NR2F2, $0.2 \mu \mathrm{g}$ of mutant NR2F2, and $0.1 \mu \mathrm{g}$ of wild-type NR2F2 $+0.1 \mu \mathrm{g}$ of mutant NR2F2), and significant statistical differences were indicated $(\mathrm{F}=42.0308, \mathrm{P}=0.00030)$. Comparisons were made between $0.2 \mu \mathrm{g}$ of wild-type NR2F2 and $0.2 \mu \mathrm{g}$ of mutant NR2F2 ( $\mathrm{t}=11.245, \mathrm{P}=0.0043)$, between $0.2 \mu \mathrm{g}$ of wild-type $\mathrm{NR} 2 \mathrm{~F} 2$ and $0.1 \mu \mathrm{g}$ of wild-type NR2F2 $+0.1 \mu \mathrm{g}$ of mutant NR2F2 ( $\mathrm{t}=5.837$, $\mathrm{P}=0.0054)$, and between $0.2 \mu \mathrm{g}$ of mutant NR2F2 and $0.1 \mu \mathrm{g}$ of wild-type $\mathrm{NR} 2 \mathrm{~F} 2+0.1 \mu \mathrm{g}$ of mutant NR2F2 (t=-7.888, $\mathrm{P}=0.0104) . \mathrm{NR} 2 \mathrm{~F} 2$, nuclear receptor subfamily 2 group F member 2; APOB, apolipoprotein B; APOB-luc, APOB promoter-driven firefly luciferase.

left coronary aortic valve cusps, and two of the six patients (II-3 and III-4) had aortic stenosis accompanied with aortic regurgitation. A total of two family members (III-1 and IV-1) of the proband had ventricular septal defect (VSD) in addition to BAV. The pedigree structure of the proband is presented in Fig. 1C. The phenotypic features and mutational status of the affected living relatives of the proband are presented in Table II. Notably, this was the only mutation detected in the present study, and no other sequence variations in $N R 2 F 2$ were detected.

Absence of transcriptional activation by the mutant $N R 2 F 2$ protein. As presented in Fig. 2, $0.2 \mu \mathrm{g}$ of wild-type

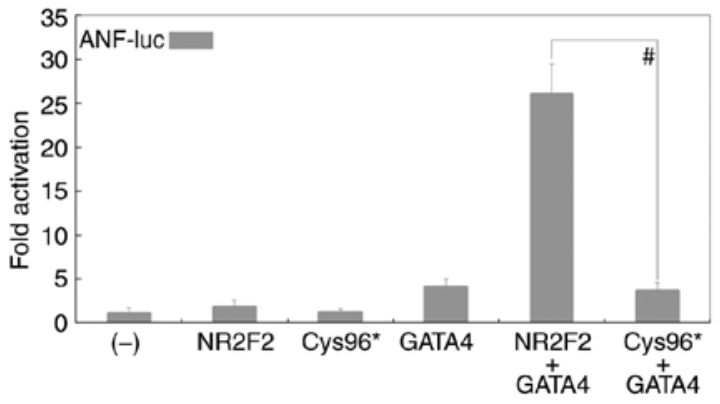

Figure 3. Disrupted synergistic transcriptional activation between mutant NR2F2 and GATA4. In the presence of GATA4, activation of the ANF promoter driven luciferase in COS-7 cells by wild-type NR2F2 or Cys96*-mutant NR2F2 (Cys96 ${ }^{*}$ ) revealed that the mutation disrupted the synergic transcriptional activation between NR2F2 and GATA4. Experiments were conducted in triplicate, and data are presented as the mean \pm standard deviation. ${ }^{\#} \mathrm{t}=11.1487, \mathrm{P}=0.00037$, compared with the wild-type counterpart. One-way analysis of variance, followed by the Fisher's protected least-squares difference post hoc test, was used for multiple comparisons (NR2F2, Cys96*, GATA4, NR2F2 + GATA4 and Cys96* + GATA4), and significant statistical differences were indicated $(\mathrm{F}=122.651, \mathrm{P}=0.0000000188)$. Comparisons were made between NR2F2 and NR2F2 + GATA4 ( $\mathrm{t}=12.221, \mathrm{P}=0.0036)$, between Cys96* and Cys96* + GATA4 $(\mathrm{t}=-4.32, \mathrm{P}=0.0318)$ between GATA4 and $\mathrm{NR} 2 \mathrm{~F} 2+$ GATA4 $(\mathrm{t}=-10.888, \mathrm{P}=0.0048)$, and between NR2F2 + GATA4 and Cys96* + GATA4 ( $\mathrm{t}=11.149, \mathrm{P}=0.0045)$. NR2F2, nuclear receptor subfamily 2 group F member 2; ANF-luc, atrial natriuretic factor promoter-driven firefly luciferase reporter plasmid.

NR2F2-pcDNA3.1 plasmid and $0.2 \mu \mathrm{g}$ of Cys96*-mutant NR2F2-pcDNA3.1 plasmid transactivated the $A P O B$ promoter in 293 cells by $\sim 13$ - and $\sim 1$-fold, respectively. When $0.1 \mu \mathrm{g}$ of wild-type NR2F2-pcDNA3.1 plasmid was utilized alone or in combination with $0.1 \mu \mathrm{g}$ of Cys96 ${ }^{*}$-mutant NR2F2-pcDNA3.1 plasmid, the resultant transactivation of the $A P O B$ promoter in COS-7 cells was $\sim 7$ - and $\sim 6$-fold, respectively. These functional results suggest that the Cys96*-mutant NR2F2 protein has no transcriptional activity.

Synergistic transactivation between NR2F2 and GATA4 disrupted by the mutation. As presented in Fig. 3, $0.1 \mu \mathrm{g}$ of wild-type NR2F2-pcDNA3.1, $0.1 \mu \mathrm{g}$ of Cys96*-mutant NR2F2-pcDNA3.1 or $0.1 \mu \mathrm{g}$ of GATA4-pSSRa transactivated the $A N F$ promoter in COS-7 cells by $\sim 2-, \sim 1-$ and $\sim 4$-fold, 
respectively. In the presence of $0.1 \mu \mathrm{g}$ of GATA4-pSSRa, the use of $0.1 \mu \mathrm{g}$ of wild-type NR2F2-pcDNA3.1 and $0.1 \mu \mathrm{g}$ of Cys96-mutant NR2F2-pcDNA3.1 transactivated the ANF promoter in 293 cells by $\sim 26$ - and $\sim 4$-fold, respectively, suggesting that the mutation disrupts the synergistic transcriptional activation between NR2F2 and GATA4.

\section{Discussion}

In the present study, a novel heterozygous mutation in the $N R 2 F 2$ gene, NM_021005.3: c.288C $>$ A; p. $($ Cys96*), was identified in a family affected with congenital BAV. The truncating mutation, which co-segregated with BAV in the family, was absent from the 560 control chromosomes. Biological measurements revealed that the Cys $96{ }^{*}$-mutant NR2F2 protein lost transactivational function. In addition, the nonsense mutation disrupted the synergistic transactivation between NR2F2 and GATA4, another cardiac core transcription factor that has been causally linked to BAV in humans $(11,18)$. Therefore, it is likely that the genetic defect of $N R 2 F 2$ contributes to BAV in this family.

In humans, $N R 2 F 2$ is located on chromosome $15 \mathrm{q} 26.2$, coding for a nuclear receptor protein with 414 amino acids, which is key to proper cardiovascular morphogenesis $(25,26)$. As shown in Fig. 1B, as a transcription factor of the nuclear receptor superfamily, NR2F2 has the typical structure of a nuclear receptor, possessing two ligand-independent transcriptional activation domains, one DNA-binding domain and one ligand-binding domain (48). The ligand-independent transcriptional activation domain at the amino terminus of the nuclear receptor is associated with ligand-independent activation, whereas the ligand-binding domain of the nuclear receptor, which contains the ligand-independent transcriptional activation domain close to the carboxyl terminus, is responsible for ligand recognition, ligand-dependent activation and receptor dimerization; the conformational state of this ligand-independent transcriptional activation domain determines the transcriptional activity of the nuclear receptor, and the DNA-binding domain is required for DNA binding, dimerization and interactions with co-factors (48). In the present study, the mutation identified in cases afflicted with BAV was anticipated to generate a truncated NR2F2 protein with only the ligand-independent transcriptional activation domain at the amino terminus and a small region of the DNA-binding domain; and this was expected to eliminate the transactivation of its target genes, encompassing $A P O B$ and $A N F(30,49)$. As hypothesized, the functional assays showed that the Cys96"-mutant NR2F2 protein failed to transactivate the $A P O B$ and $A N F$ promoters, alone or synergistically with GATA4. These data suggested that $N R 2 F 2$ haploinsufficiency caused by the nonsense mutation may be a molecular mechanism underlying BAV.

Notably, the mutation was firstly identified in only one case among 176 patients, suggesting it is not a common mutation. However, the mutation was identified in all affected members of a family, but was absent in unaffected family members and the 280 controls. Furthermore, the mutation has not been reported previously, and functional analysis revealed that the mutation nullified the transcription activity of NR2F2 alone or in synergy with GATA4. These are important novel findings.
However, western blot analysis can show a truncated size of NR2F2, which may be more convincing in supporting the conclusion.

An association between genetically compromised $N R 2 F 2$ and enhanced susceptibility to BAV may be attributable to abnormal cardiac valvular morphogenesis. In mice, the NR2F2 protein was shown to be expressed at high levels in the atria and ventricles throughout embryogenesis. Particularly by E9.5, an abundant expression of $\mathrm{NR} 2 \mathrm{~F} 2$ was observed in the endocardium and epicardium of the ventricle, the endocardium of the atrioventricular cushion, and the endocardium and myocardium of the atrium $(25,26)$. In the mice, knockout of the $N r 2 f 2$ gene resulted in embryonic death due to defects in angiogenesis and cardiogenesis (25). In mice with endothelial-specific disruption of $N r 2 f 2$, multiple cardiovascular morphogenic anomalies occurred, encompassing atrioventricular septal defect, hypoplastic ventricles and hypoplasia of endocardial cushions (26). The endocardial cushion hypoplasia caused by $N r 2 f 2$ deficiency was ascribed to the decreased growth and survival of mesenchymal cells from the atrioventricular cushion and impaired epithelial-mesenchymal transformation in the endocardium (26). Notably, the impairment in endocardial epithelial-mesenchymal transformation was accompanied by the downregulation of snail family transcriptional repressor 1 , a master regulator of epithelial-mesenchymal transformation (26). In humans, NR2F2 has been shown to be expressed at a high level in the embryonic heart, encompassing the atria, aorta and coronary vessels, and pathogenic mutations in the $N R 2 F 2$ gene have been reported to cause various cardiovascular abnormalities, including atrioventricular septal defect, VSD, double outlet right ventricle, coarctation of the aorta, aortic stenosis, tetralogy of Fallot and hypoplastic left heart syndrome (30-33). In the present study, a novel $N R 2 F 2$ loss-of-function mutation was associated with BAV, in addition to VSD, which expands on the $N R 2 F 2$-associated phenotypic spectrum. Collectively, the present data provided novel insight into the pivotal role of NR2F2 in cardiovascular development, particularly in the development of the endocardium for endocardial epithelial-mesenchymal transformation, which is crucial for normal valve formation.

In conclusion, the present study identified $N R 2 F 2$ as a novel susceptibility gene for BAV in humans, providing novel insight into the genetic mechanism underlying BAV, and indicating potential significance for genetic counseling and individualized treatment for cases affected with BAV.

\section{Acknowledgements}

Not applicable.

\section{Funding}

The present study was financially supported in part by grants from the National Natural Science Foundation of China (grant nos. 81641014, 81470372, 81400244, 81370400 and 81271927), the Clinical Research Plan of SHDC, Shanghai, China (grant no. 16CR3005A), the Medicine Guided Program of Shanghai, China (grant no. 17411971000), 
the Experimental Animal Program of Shanghai, China (grant no. 17140902400) and the Fundamental Research Funds for the Central Universities. The funding bodies had no involvement in the design of the study, collection, analysis or interpretation of data, or manuscript writing.

\section{Availability of data and materials}

All data generated or analyzed during this study are included in this published article.

\section{Authors' contributions}

JW and YQY conceived and designed the study, and were major contributors in writing the manuscript. JW, PA, YJX, RGL, MZ, XBQ, RMD, QQ, XML, RTH and SX analyzed and interpreted the patient data. JW, PA, YJX and YQY performed the molecular and biochemical experiments. All authors read and approved the final manuscript.

\section{Ethics approval and consent to participate}

This study was performed in accordance with the 1964 Helsinki declaration and its later amendments. The study protocol was approved by the Medical Ethics Committee of East Hospital, Tongji University School of Medicine, China. The study subjects provided written informed consent to participate in the study.

\section{Patient consent for publication}

The patients provided written informed consent for the publication of any associated data.

\section{Competing interests}

The authors declare that they have no competing interests.

\section{References}

1. Gago-Díaz M, Brion M, Gallego P, Calvo F, Robledo-Carmona J, Saura D, Sánchez V, Bermejo J, Sevilla T, Newton-Cheh C, et al: The genetic component of bicuspid aortic valve and aortic dilation. An exome-wide association study. J Mol Cell Cardiol 102: 3-9, 2017.

2. Li Y, Wei X, Zhao Z, Liao Y, He J, Xiong T, Xu Y, Lv W, Ou Y, Tang $\mathrm{H}$, et al: Prevalence and complications of bicuspid aortic valve in Chinese according to echocardiographic database. Am J Cardiol 120: 287-291, 2017.

3. Roberts WC, Janning KG, Vowels TJ, Ko JM, Hamman BL and Hebeler RF Jr: Presence of a congenitally bicuspid aortic valve among patients having combined mitral and aortic valve replacement. Am J Cardiol 109: 263-271, 2012.

4. Prakash SK, Bossé Y, Muehlschlegel JD, Michelena HI, Limongelli G, Della Corte A, Pluchinotta FR, Russo MG, Evangelista A, Benson DW, et al: A roadmap to investigate the genetic basis of bicuspid aortic valve and its complications: Insights from the International BAVCon (Bicuspid Aortic Valve Consortium). J Am Coll Cardiol 64: 832-839, 2014.

5. Girdauskas E and Petersen J: Update on bicuspid aortic valve aortopathy. Curr Opin Cardiol 32: 651-654, 2017.

6. Kong WKF, Regeer MV, Poh KK, Yip JW, van Rosendael PJ, Yeo TC, Tay E, Kamperidis V, van der Velde ET, Mertens B, et al Inter-ethnic differences in valve morphology, valvular dysfunction, and aortopathy between Asian and European patients with bicuspid aortic valve. Eur Heart J 39: 1308-1313, 2018.
7. Zegri-Reiriz I, de Alarcón A, Muñoz P, Martínez Sellés M, González-Ramallo V, Miro JM, Falces C, Gonzalez Rico C, Kortajarena Urkola X, Lepe JA, et al: Infective endocarditis in patients with bicuspid aortic valve or mitral valve prolapse. J Am Coll Cardiol 71: 2731-2740, 2018.

8. Lluri G, Renella P, Finn JP, Vorobiof G, Aboulhosn J and Deb A: Prognostic significance of left ventricular fibrosis in patients with congenital bicuspid aortic valve. Am J Cardiol 120: 1176-1179, 2017.

9. Masri A, Svensson LG, Griffin BP and Desai MY: Contemporary natural history of bicuspid aortic valve disease: A systematic review. Heart 103: 1323-1330, 2017.

10. Padang R, Bagnall RD and Semsarian C: Genetic basis of familial valvular heart disease. Circ Cardiovasc Genet 5: 569-580, 2012.

11. Yang B, Zhou W, Jiao J, Nielsen JB, Mathis MR, Heydarpour M, Lettre G, Folkersen L, Prakash S, Schurmann C, et al: Protein-altering and regulatory genetic variants near GATA4 implicated in bicuspid aortic valve. Nat Commun 8: 15481, 2017.

12. Pan S, Lai H, Shen Y, Breeze C, Beck S, Hong T, Wang C and Teschendorff AE: DNA methylome analysis reveals distinct epigenetic patterns of ascending aortic dissection and bicuspid aortic valve. Cardiovasc Res 113: 692-704, 2017.

13. Giusti B, Sticchi E, De Cario R, Magi A, Nistri S and Pepe G: Genetic bases of bicuspid aortic valve: The contribution of traditional and high-throughput sequencing approaches on research and diagnosis. Front Physiol 8: 612, 2017.

14. Garg V, Muth AN, Ransom JF, Schluterman MK, Barnes R, King IN, Grossfeld PD and Srivastava D: Mutations in NOTCH1 cause aortic valve disease. Nature 437: 270-274, 2005.

15. Dargis N, Lamontagne M, Gaudreault N, Sbarra L, Henry C, Pibarot $\mathrm{P}$, Mathieu $\mathrm{P}$ and Bossé Y: Identification of gender-specific genetic variants in patients with bicuspid aortic valve. Am J Cardiol 117: 420-426, 2016

16. Gillis E, Kumar AA, Luyckx I, Preuss C, Cannaerts E, van de Beek $G$, Wieschendorf B, Alaerts M, Bolar N, Vandeweyer $\mathrm{G}$, et al: Candidate gene resequencing in a large bicuspid aortic valve-associated thoracic aortic aneurysm cohort: SMAD6 as an important contributor. Front Physiol 8: 400, 2017.

17. Lin X, Liu X, Wang L, Jiang J, Sun Y, Zhu Q, Chen Z, He Y, Hu P, Xu Q, et al: Targeted next-generation sequencing identified ADAMTS5 as novel genetic substrate in patients with bicuspid aortic valve. Int J Cardiol 252: 150-155, 2018.

18. Li RG, Xu YJ, Wang J, Liu XY, Yuan F, Huang RT, Xue S, Li L, Liu H, Li YJ, et al: GATA4 loss-of-function mutation and the congenitally bicuspid aortic valve. Am J Cardiol 121: 469-474, 2018.

19. Padang R, Bagnall RD, Richmond DR, Bannon PG and Semsarian C: Rare non-synonymous variations in the transcriptional activation domains of GATA5 in bicuspid aortic valve disease. J Mol Cell Cardiol 53: 277-281, 2012.

20. Bonachea EM, Chang SW, Zender G,LaHaye S, Fitzgerald-Butt S, McBride KL and Garg V: Rare GATA5 sequence variants identified in individuals with bicuspid aortic valve. Pediatr Res 76: 211-216, 2014.

21. Shi LM, Tao JW, Qiu XB, Wang J, Yuan F, Xu L, Liu H, Li RG, $\mathrm{Xu}$ YJ, Wang Q, et al: GATA5 loss-of-function mutations associated with congenital bicuspid aortic valve. Int J Mol Med 33: 1219-1226, 2014

22. Xu YJ, Di RM, Qiao Q, Li XM, Huang RT, Xue S, Liu XY, Wang J and Yang YQ: GATA6 loss-of-function mutation contributes to congenital bicuspid aortic valve. Gene 663: 115-120, 2018.

23. Gharibeh L, Komati H, Bossé Y, Boodhwani M, Heydarpour M, Fortier M, Hassandazeh R, Ngu J, Mathieu P, Body S, et al: GATA6 regulates aortic valve remodeling and its haploinsufficiency leads to RL-type bicuspid aortic valve. Circulation 138: 1025-1038, 2018.

24. Qu XK, Qiu XB, Yuan F, Wang J, Zhao CM, Liu XY, Zhang XL, Li RG, Xu YJ, Hou XM, et al: A novel NKX2.5 loss-of-function mutation associated with congenital bicuspid aortic valve. Am J Cardiol 114: 1891-1895, 2014.

25. Pereira FA, Qiu Y, Zhou G, Tsai MJ and Tsai SY: The orphan nuclear receptor COUP-TFII is required for angiogenesis and heart development. Genes Dev 13: 1037-1049, 1999.

26. Lin FJ, You LR, Yu CT, Hsu WH, Tsai MJ and Tsai SY: Endocardial cushion morphogenesis and coronary vessel development require chicken ovalbumin upstream promoter-transcription factor II. Arterioscler Thromb Vasc Biol 32: e135-e146, 2012.

27. Pikkarainen S, Tokola H, Kerkelä R and Ruskoaho H: GATA transcription factors in the developing and adult heart. Cardiovasc Res 63: 196-207, 2004. 
28. Lentjes MH, Niessen HE, Akiyama Y, de Bruïne AP, Melotte V and van Engeland M: The emerging role of GATA transcription factors in development and disease. Expert Rev Mol Med 18: e3, 2016.

29. Akazawa $\mathrm{H}$ and Komuro I: Cardiac transcription factor $\mathrm{Csx} / \mathrm{Nkx} 2-5$ : Its role in cardiac development and diseases. Pharmacol Ther 107: 252-268, 2005.

30. Al Turki S, Manickaraj AK, Mercer CL, Gerety SS, Hitz MP, Lindsay S, D'Alessandro LCA, Swaminathan GJ, Bentham J, Arndt AK, et al: Rare variants in NR2F2 cause congenital heart defects in humans. Am J Hum Genet 94: 574-585, 2014

31. Qiao XH, Wang Q, Wang J, Liu XY, Xu YJ, Huang RT, Xue S, Li YJ, Zhang M, Qu XK, et al: A novel NR2F2 loss-of-function mutation predisposes to congenital heart defect. Eur J Med Genet 61: 197-203, 2018.

32. Bashamboo A, Eozenou C, Jorgensen A, Bignon-Topalovic J, Siffroi JP, Hyon C, Tar A, Nagy P, Sólyom J, Halász Z, et al: Loss of function of the nuclear receptor NR2F2, encoding COUP-TF2, causes testis development and cardiac defects in 46, XX children. Am J Hum Genet 102: 487-493, 2018.

33. Upadia J, Gonzales PR and Robin NH: Novel de novo pathogenic variant in the NR2F2 gene in a boy with congenital heart defect and dysmorphic features. Am J Med Genet A 176: 1423-1426, 2018.

34. Liu Y, Li B, Xu Y and Sun K: Mutation screening of GATA4 gene in CTD patients within Chinese Han population. Pediatr Cardiol 38: 506-512, 2017.

35. Yu Y, Lei W, Yang J, Wei YC, Zhao ZL, Zhao ZA and Hu S Functional mutant GATA4 identification and potential application in preimplantation diagnosis of congenital heart diseases. Gene 641: 349-354, 2018

36. Hempel M, Casar Tena T, Diehl T, Burczyk MS, Strom TM, Kubisch C, Philipp M and Lessel D: Compound heterozygous GATA5 mutations in a girl with hydrops fetalis, congenital heart defects and genital anomalies. Hum Genet 136: 339-346, 2017.

37. Zhang E, Hong N, Chen S, Fu Q, Li F, Yu Y and Sun K: Targeted sequencing identifies novel GATA6 variants in a large cohort of patients with conotruncal heart defects. Gene 641: 341-348, 2018.

38. Xu YJ, Qiu XB, Yuan F, Shi HY, Xu L, Hou XM, Qu XK, Liu X, Huang RT, Xue S, et al: Prevalence and spectrum of NKX2.5 mutations in patients with congenital atrial septal defect and atrioventricular block. Mol Med Rep 15: 2247-2254, 2017.
39. Zaidi S and Brueckner M: Genetics and genomics of congenital heart disease. Circ Res 120: 923-940, 2017.

40. Li YJ and Yang YQ: An update on the molecular diagnosis of congenital heart disease: Focus on loss-of-function mutations. Expert Rev Mol Diagn 17: 393-401, 2017.

41. Li N, Wang ZS, Wang XH, Xu YJ, Qiao Q, Li XM, Di RM, Guo XJ, Li RG, Zhang M, et al: A SHOX2 loss-of-function mutation underlying familial atrial fibrillation. Int J Med Sci 15: 1564-1572, 2018

42. Qiao XH, Wang F, Zhang XL, Huang RT, Xue S, Wang J, Qiu XB, Liu XY and Yang YQ: MEF2C loss-of-function mutation contributes to congenital heart defects. Int J Med Sci 14: 1143-1153, 2017.

43. Huang RT, Wang J, Xue S, Qiu XB, Shi HY, Li RG, Qu XK, Yang XX, Liu H, Li N, et al: TBX20 loss-of-function mutation responsible for familial tetralogy of Fallot or sporadic persistent truncus arteriosus. Int J Med Sci 14: 323-332, 2017.

44. Lu CX, Gong HR, Liu XY, Wang J,Zhao CM, Huang RT, Xue S and Yang YQ: A novel HAND2 loss-of-function mutation responsible for tetralogy of Fallot. Int J Mol Med 37: 445-451, 2016.

45. Qiu XB, Qu XK, Li RG, Liu H, Xu YJ, Zhang M, Shi HY, Hou XM, Liu X, Yuan F, et al: CASZ1 loss-of-function mutation contributes to familial dilated cardiomyopathy. Clin Chem Lab Med 55: 1417-1425, 2017.

46. Li L, Wang J, Liu XY, Liu H, Shi HY, Yang XX, Li N, Li YJ, Huang RT, Xue S, et al: HAND1 loss-of-function mutation contributes to congenital double outlet right ventricle. Int J Mol Med 39: 711-718, 2017.

47. Yuan F, Qiu ZH, Wang XH, Sun YM, Wang J, Li RG, Liu H, Zhang M, Shi HY, Zhao L, et al: MEF2C loss-of-function mutation associated with familial dilated cardiomyopathy. Clin Chem Lab Med 56: 502-511, 2018.

48. Yamazaki T, Suehiro J, Miyazaki H, Minami T, Kodama T, Miyazono $\mathrm{K}$ and Watabe T: The COUP-TFII variant lacking a DNA-binding domain inhibits the activation of the Cyp7a1 promoter through physical interaction with COUP-TFII. Biochem J 452: 345-357, 2013.

49. Huggins GS, Bacani CJ, Boltax J, Aikawa R and Leiden JM: Friend of GATA 2 physically interacts with chicken ovalbumin upstream promoter-TF2 (COUP-TF2) and COUP-TF3 and represses COUP-TF2-dependent activation of the atrial natriuretic factor promoter. J Biol Chem 276: 28029-28036, 2001. 